Currículo sem Fronteiras, v. 19, n. 3, p. 1202-1224, set./dez. 2019

\title{
OS PARADOXOS DA EDUCAÇÃO PARA CIDADANIA GLOBAL NA FORMAÇÃO DOCENTE
}

\author{
Gustavo E. Fischman \\ Arizona State University \\ Marta Estellés \\ Universidade de Cantabria
}

\begin{abstract}
Resumo
Propostas de Educação para Cidadania Global (ECG) são frequentemente apresentadas como se derivaram de processos quase-naturais de evolução pedagógica. Nesse pensamento evolutivo, a ECG representa um estágio mais avançado de inovação pedagógica e, consequentemente, aparece como o melhor e mais abrangente modelo educacional. É importante apontar que no período 19902019 duas tendências simultâneas procuram desenvolver modelos de cidadania diferentes. Se a ECG procura desenvolver um/a cidadão global cosmopolita outras propostas pedagógicas apontam a transformar as noções de cidadania dando prioridade aos direitos de um "consumidor global" neoliberal sobre os direitos "cidadãos globais/cívicos". Este artigo discute 62 artigos sobre ECG e identifico o predomínio de uma orientação pedagógica redentora idealizada que tem muitas limitações e fundamentalmente não confronta às opções consumistas oferecidas pelos modelos pedagógicos de orientação neoliberais.
\end{abstract}

Palavras-chave: Educação para Cidadania Global; formação docente; pedagogias redentoras, Movimentos Globais de Reforma.

\begin{abstract}
Proposals for Global Citizenship Education (ECG) are often presented as derived from quasi-natural processes of pedagogical evolution. In this evolutionary thinking, ECG represents a more advanced stage of pedagogical innovation and, consequently, appears as the best and most comprehensive educational model. It is important to point out that in the period 1990-2019 two simultaneous trends seek to develop different models of citizenship. If the ECG seeks to develop a cosmopolitan global citizen, other pedagogical proposals aim to transform notions of citizenship by giving priority to the rights of a neoliberal "global consumer" over "global / civic" rights. This article reviewed 62 peerreviewed articles on ECG and identified the predominance of an idealized redemptive pedagogical orientation that has many limitations and fundamentally does not confront the consumerist options offered by neoliberal pedagogical models.
\end{abstract}

Keywords: Global Citizenship Education; teacher education, redemptive pedagogies; Global Reform Movements.

ISSN 1645-1384 (online) www.curriculosemfronteiras.org 


\section{A educação para cidadania global e as reformas globais}

Nos anos de 1990 - e com mais força no início do século XXI - eclodiram séries de políticas educativas e projetos relacionados à educação para a cidadania global. Um dos pressupostos centrais da educação cidadã global é que os modelos de cidadania nacional já não são adequados para os novos cenários e subjetividades globais (BAUMAN, 2001; DALE \& ROBERTSON, 2007; ROBERTSON \& DALE, 2008). Como Enslin (2000, p. 149) assinala,

[...] a educação institucionalizada baseada na identidade definida pela participação em uma 'nação' repousa na suposição equivocada de que a democracia é efetivamente buscada dentro do Estado-nação, cuja influência e autoridade foi reduzida pela globalização.

Embora alguns defensores da educação para cidadania global pareçam ingenuamente ignorar que os cidadãos só exercem direitos e responsabilidades de cidadania plena em nível local e nacional (MARSHALL, 2011) ${ }^{1}$, as propostas de educação cidadã global desafiam indubitavelmente as formas de educação cívica restritas aos discursos nacionalistas tradicionais que, por sua vez, muitas vezes promoviam noções de patriotismos ritualizados e subordinação dos cidadãos à autoridade do Estado.

Ainda que a presente discussão não se debruce sobre o enfraquecimento mundial dos modelos políticos de cidadania nacional ${ }^{2}$, é importante reconhecer que, como conceito, a cidadania não é uma noção estática, fixa e, muito menos, uma ideia ou um ideal de fácil definição (BENHABIB, 2005; 2007). Os persistentes debates sobre como lidar com a situação migratória dos milhões de deslocados pela guerra e pela pobreza endêmica, evidenciam que em estados democráticos as pessoas, em tese, decidem quem se qualifica ou não para a cidadania. Sobre esse movimento Benhabib (2005, p. 66) afirma:

[...] hoje somos apanhados não só na reconfiguração da soberania, mas também nas reconstituições da cidadania. Estamos nos afastando da cidadania como membros nacionais cada vez mais para uma cidadania de residência, o que fortalece os múltiplos laços com a localidade, com a região e com as instituições transnacionais ${ }^{3}$.

Neste artigo, analisamos os discursos que moldam tacitamente os significados da Educação para a Cidadania Global (ECG) na literatura sobre formação de professores para identificar continuidades e mudanças conceituais nas perspectivas da educação para a cidadania. Mais especificamente, este artigo tem dois objetivos centrais: a) discutir as propostas pedagógicas apresentadas em 62 artigos sobre educação para a cidadania global, publicados em inglês durante o período 1990-2018; e b) analisar as propostas pedagógicas da ECG, procurando entende-las em relação à crescente consolidação da narrativa do que Lindgard e Sahlberg (LINDGARD et al., 2013; SAHLBERG, 2010) denominam como Movimento Global de Reforma Educativa. O Movimento Global da Reforma Educativa 
(MGRE) é caracterizado por uma forte defesa das opções privadas, pela diminuição do setor público na educação e pela promoção de transações comerciais na seleção de serviços educacionais. Além disso, o MGRE se caracteriza pelo uso generalizado de testes padronizados para avaliar a qualidade acadêmica e por responsabilizar única e exclusivamente os professores pelos resultados acadêmicos dos estudantes. Essas medidas se conjugam com a forte promoção da privatização e comercialização da educação, bem como à redução do papel dos sindicatos de professores e da regulação estatal da educação.

É importante explicitar que compreendemos que a maioria dos pesquisadores está ciente de que a ECG não é um conceito neutro e que as tipologias sobre os modelos ideais de cidadania global são abundantes na literatura educacional (GOREN \& YEMINI, 2017). Em vez de produzir mais uma tipologia, analisamos esses entendimentos tácitos que emolduram e idealizam a ECG dentro da literatura sobre formação docente e as suas conexões com os movimentos de reforma educativa global.

\section{Tendências na literatura: Educação para a Cidadania Global na formação docente}

Talvez mais do que nunca, os professores de hoje devam equipar os alunos com o conhecimento, valores, atitudes e habilidades necessárias para ter sucesso em uma sociedade cada vez mais globalizada (CUTURARA, 2009; EVANS 2006; MUNDY \& MANION 2008; SMITH, 2006; WAR CHILD CANADA, 2006). Aceitando esse desafio, os proponentes da Educação para a Cidadania Global buscam desenvolver os conhecimentos e as capacidades dos estudantes para participar ativamente como cidadãos globais, com o objetivo final de criar um mundo mais justo, pacífico e democrático (BLANEY, 2002; GALLAVAN, 2008; GARRATT \& PIPER, 2003; HICKS \& BORD, 2001; MUNDY \& MANION, 2008; TROTTA TUOMI, 2004). Dado o peso da responsabilidade atribuída aos professores de hoje, essas questões merecem uma análise cuidadosa para informar o desenvolvimento profissional em serviço dos educadores (APPLEYARD e MCLEAN, 2011, p. 6).

O raciocínio acima citado é notavelmente comum na literatura sobre Educação para a Cidadania Global (ECG) relacionada com as licenciaturas ${ }^{4}$. A literatura analisada tinha também um forte interesse em tópicos como educação global, educação internacional, educação em direitos humanos, educação para o desenvolvimento, educação ambiental, educação para a paz e educação intercultural, dentre outras configurações.

Davies e colaboradores (2005) situam o surgimento da ECG na convergência explícita de duas correntes: a "educação para a cidadania" e a "educação global" abrangente de "educação global" anteriormente unificou outros modelos educacionais focados em questões globais, como a educação em direitos humanos, a educação para o desenvolvimento, a educação ambiental e a educação para a paz (PIKE, 2008), a ECG é resultado da integração de todas as tendências da educação global e das perspectivas da educação para a cidadania, o que significa, em suma, abraçar noções de cidadania que reconhecem direitos e deveres para além das fronteiras do estado-nação (DAVIES, 2006). 
A ECG é frequentemente apresentada como o resultado de um modelo pedagógico evolucionário simples, ou seja, o modelo mais recente, melhor e mais abrangente que incorpora todos os objetivos e as práticas positivas de esforços anteriores, como as acima mencionadas categorias de educação - direitos humanos, cidadania, multiculturalismo, global, ambiental e para a paz - e supera suas limitações. Nesse sentido, podemos mencionar como exemplos as ideias de Hahn (2005), que defende a ECG porque a educação em direitos humanos, embora essencial, nem sempre é suficiente; Davies, Evans e Reid (2005), que criticam as limitações da "educação global" e da "educação para a cidadania", mas, percebendo suas potencialidades, apoiam a integração de ambos modelos na ECG; Mannion et al. (2011), que defendem a ECG porque ela reúne "educação ambiental”, "educação para o desenvolvimento" e "educação para a cidadania"; Su, Bullivant e Holt (2013), que concebem a ECG como o resultado do desenvolvimento e convergência da "educação global”, da "educação para o desenvolvimento" e da "educação para a cidadania”; de Eidoo et al. (2011), que conceituam ECG “[...] como uma extensão natural à educação multicultural" (p. 67); e, finalmente, o programa de desenvolvimento profissional em ECG tal como descrito por Appleyard e McLean (2011, p.10): seu objetivo é, seguindo os autores, integrar "[...] os temas de paz e justiça, direitos humanos, sustentabilidade ambiental e desenvolvimento internacional nos currículos e práticas educacionais". A aprovação desse modelo foi tão consensual e difundida que Yemini (2017) descreveu o termo "ECG" como uma palavra de ordem. A isso, somamos o que outros autores chamaram de termo "guardachuva", considerando a ampla gama de perspectivas e temas que esse modelo integrou (DAVIES, 2006; JORGENSON \& SHULTZ, 2012).

Sabemos que ser capaz de identificar as fraquezas dos modelos tradicionais não é o mesmo que superar as limitações desses modelos de educação para a cidadania. Pensemos, por exemplo, na noção de "cidadão" disposta na maioria dos programas de educação para cidadania. Essa noção é frequentemente baseada em um cidadão cartesiano desencarnado (FISCHMAN \& HAAS, 2012), ou seja, um sujeito idealizado, cujos comportamentos políticos são o efeito direto de processos racionais desencarnados que não reconhecem o grande papel das emoções ou paixões na tomada de decisões ${ }^{6}$. Em sua contraposição, modos de pensar encarnados inconscientes e emocionais desempenham um papel fundamental em nossas ações e percepções diárias. Os cidadãos, sejam globais ou nacionais, não são desapaixonados (MARCUS, 2002). Entretanto, um imaginário que associa a noção de cidadão com figuras heroicas e românticas é dominante. Os exemplos a seguir capturam o romantismo inerente a esse conceito de um cidadão descarnado e idealizado que domina a maior parte da literatura sobre a $\mathrm{ECG}^{7}$ :

Uma imagem, então, do cidadão global: não apenas consciente de seus direitos, mas capaz e desejoso de agir sobre eles; de uma disposição crítica autônoma e inquisitiva; mas suas decisões e ações são temperadas por uma preocupação ética pela justiça social e pela dignidade da humanidade; portanto, capaz, através de suas ações, de controlar e melhorar a "trajetória do eu" através da vida enquanto contribui para o 
bem comum, o bem público, com um senso de dever cívico de reabastecer a sociedade (GRIFFITHS, 1998, p. 40).

Respeito pelos semelhantes, independentemente de raça, sexo, idade, religião ou opiniões políticas; apreço pela diversidade e múltiplas perspectivas; uma visão de que nenhuma sociedade ou cultura é inerentemente superior a qualquer outra; apreço pelo mundo natural e respeito pelos direitos de todos os seres vivos; prática e encorajamento de padrões sustentáveis de vida, consumo e produção; esforço por resolver conflitos sem o uso de violência; ser responsável por resolver os desafios globais prementes de todas as formas possíveis; pensar globalmente e agir localmente na erradicação da desigualdade e da injustiça em todas as suas formas (GUO, 2014, p. 2).

O retrato feito por Guo sobre o cidadão global pressupõe que qualquer cidadão age de forma consistente, de acordo com ideias e valores pressupostos nos modelos de racionalidade do homo-economicus ou homo-politicus. Nesse sentido, para os defensores do ECG, seria necessária a fabricação de um novo tipo de educador profissional, que compartilhasse a tradição do professor romantizado, idealizado e cujos comportamentos políticos fossem honestos, desinteressados e emergentes de processos cognitivos desencarnados e deliberados autônomos ${ }^{8}$. A presença dessa visão do cidadão como ator político desencarnado e puramente racional nos discursos da ECG é apenas um exemplo de um ponto fraco desse modelo aparentemente perfeito. A tradição cartesiana de cogito, ergo sum ignora a importância da emoção e das experiências vividas na aprendizagem cívica, resultando em modelos excessivamente impraticáveis (FISCHMAN \& HAAS, 2014; KNIGHT ABOWITZ, 2008; SCHUGURENSKY, 2010). Isso acontece mesmo com as propostas de pedagogia ECG que promovem programas interculturais de intercâmbio e estudo no exterior (ROBERTS, 2007), considerando essas experiências suficientes para obter professores globalmente competentes (PARKHOUSE, et al., 2016; TRILOKEKAR \& KUKAR, 2011). Outra crítica frequente e pertinente ao ECG é seu viés cultural ocidental (ANDREOTTI \& DE SOUZA, 2012; DILL, 2015; HANDLER, 2013; HARTMAN \& KIELY, 2014; JEFFRESS, 2012; WANG \& HOFFMAN, 2016). É, portanto, muito questionável considerar o ECG como o modelo mais evoluído para a educação. Tal consideração faz pleno sentido apenas quando compõe um discurso romantizado que aponta para a educação como redentora, como explicaremos no próximo tópico. 


\section{Educação para a Cidadania Global: uma solução redentora para problemas globais não educacionais?}

As propostas do ECG analisadas foram produzidas usando modelos conceituais muito diferentes, sem consenso sobre o que de fato significa a cidadania global ${ }^{9}$ (GAUDELLI, 2016; JORGENSON \& SHULTZ, 2012; NODDINGS, 2005; SHULTZ, 2007). Alguns autores enfatizam a importância dos direitos humanos (OSLER, 2005), conectando essa área com a antiga tradição estoica do cosmopolitismo (HEATER, 2004; NUSSBAUM, 1997), enquanto outros enfatizam a participação dos estudantes na governança global (PIKE, 2008), considerando a falta de controle democrático e regulação da globalização (ARCHIBUGI \& HELD, 2011; HELD, 1997, 2010). Alguns autores se concentram no discurso de defesa do desenvolvimento de um sentimento de pertencer a uma comunidade global (DE RIVERA \& CARSON, 2015), enquanto outros destacam a consciência crítica sobre as assimetrias globais de poder (ARNOLD, 2014; CAMICIA \& FRANKLIN, 2011).

Talvez a única tendência implícita unificadora dessas ideias seja o reconhecimento da existência de um novo cenário geopolítico e, por consequência, um senso de urgência para responder aos desafios advindos da globalização (STROMQUIST \& MONKMAN, 2014; SUÁREZ-OROZCO, 2007). A maioria das políticas da ECG e documentos institucionais exaltam a relevância de incluir a ECG nos currículos nacionais para enfrentar os desafios globais, preparando os estudantes para se tornarem "[...] contribuintes proativos para um mundo mais justo, pacífico, tolerante, inclusivo, seguro e sustentável” (UNESCO, 2013, p. 3). Os problemas globais comumente referidos pelas políticas nacionais da ECG são geralmente relacionados à resolução de conflitos, ao desenvolvimento sustentável, ao respeito pelos direitos humanos e pela diversidade, à interdependência econômica, e às desigualdades sociais (MANNION et al., 2011) ${ }^{10}$. Evidentemente, essa tendência também está presente em muitos programas docentes, como Guo (2014, p. 2) afirma em sua defesa para a implantação de ECG em cursos de formação de professores:

Os estudantes de hoje estão se formando em um mundo que está interconectado como nunca. Como cidadãos no século 21, eles são obrigados a ser responsáveis e responsivos à miríade de problemas complexos e questões de interesse global e local, seja na saúde, meio ambiente, paz ou segurança econômica. Esse contexto global em mutação exige que os estudantes desenvolvam hoje o conhecimento, as habilidades, os atributos e o compromisso com a cidadania global por meio do processo educacional.

Ao abraçar a ideia de que "[...] a ECG visa capacitar os alunos para se envolverem e assumirem papéis ativos, tanto local quanto globalmente, para enfrentar e resolver os desafios globais" (UNESCO, 2014, p. 15), é implicitamente assumido a ênfase de que a responsabilidade de resolver esses problemas reside nos indivíduos e não em governos ou organizações internacionais. Obviamente, questões como a resolução de guerras, as mudanças climáticas, as disparidades sociais, a fome - apenas para citar algumas - não 
dependem apenas da competência de cidadania global dos indivíduos e de sua cooperação (HELD, 2016). E, mesmo assim, não é a primeira vez que governos e organizações internacionais promovem medidas curriculares e cívicas para abordar problemas sociais e políticos, ignorando ou minimizando a incidência de outras razões estruturais (EVANS, 2015; GIMENO, 2009; ROMERO \& ESTELLÉS, 2015). Essa concepção idealizada de educação, que parece apontar com predominância para o papel do indivíduo, tende a reforçar a perspectiva neoliberal de minimizar a esfera pública e as obrigações dos governos para com seus cidadãos (EVANS, 2015; ROMERO \& ESTELLÉS, 2015).

Assim, em vez de questionar essa implicação, os programas ECG tendem a exaltar a narrativa neoliberal. Outro exemplo disso pode ser encontrado no ACT! Active Citizen Today, projeto canadense para educadores de cidadania global, que tem o seguinte objetivo:

[...] apoiar os educadores de cidadania global a ensinar os alunos a assumir um papel ativo no enfrentamento da injustiça e da desigualdade, tanto local quanto globalmente. A este respeito, o projeto de kit de ensino do ACT! reconhece o potencial e a responsabilidade de professores e alunos como cidadãos globais e agentes de mudança em um mundo interconectado e interdependente (LARSEN \& FADEN, 2008, p. 74).

Uma grande parte desses programas é dedicada a enriquecer as conceituações de cidadania global dos professores, baseando-se na percepção de que eles têm uma consciência limitada sobre serem cidadãos globais (BYKER, 2016; DUCKWORTH, Levy \& LEVY, 2005; LONGVIEW FOUNDATION, 2008); e ZONG, 2009) e, por isso, dificilmente utilizam o conceito de cidadania global em suas propostas e em salas de aula (RAPOPORT, 2015). Por exemplo, um dos objetivos do projeto ECG defendido por McLean, Cook e Crowe (2006, p.04) é “[...] expandir a compreensão dos candidatos a professores sobre a cidadania global”. Da mesma forma, An (2014, p.27) explica que seu “[...] objetivo como educadora de professores é introduzir a natureza múltipla e contestada da cidadania global aos candidatos a professores e desafiá-los a refletir sobre suas próprias noções de cidadania global”. Na mesma direção, o curso de formação de professores analisado por Guo (2014), os três primeiros dos nove tópicos desenvolvidos foram: 1) Introdução à cidadania global e educação para a cidadania global; 2) Objetivos e objetivos da educação para a cidadania global e 3) Conceitos e temas chave na educação global do cidadão. Tal abordagem colocase como crucial para a configuração de um cidadão global. Essa suposição também pode ter orientado a pesquisa sobre a pedagogia ECG considerando a atenção que foi dada às percepções dos professores em serviço sobre a cidadania global (por exemplo: CARR, PLUIM, \& HOWARD, 2014; GÜNEL \& PEHLIVAN, 2016; MYERS, 2008; RAPOPORT, 2010; ROBBINS, FRANCIS \& ELLIOT, 2003).

O programa de pedagogia ECG preconizado por Blanks (2013, p. 13) nos EUA buscava, "[...] incentivar nos estudantes a crença de que eles podem fazer a diferença no mundo, para melhor, foram os resultados desejados mais importantes.” Os professores após esse workshop identificaram "[...] aumento do otimismo e da inspiração nas suas capacidades de 
transformação”. Diante dessas afirmações, pode-se inferir que o autor considera a ECG como um fator-chave de um discurso educativamente redentor.

Outra simplificação implícita a essa defesa idealizada da ECG é que ela pode resolver problemas globais, mas se constatado que ela não está solucionando os problemas de sua esfera é porque - e talvez tão somente - não esteja sendo bem implementada, cabendo ressaltar que o professor é o principal agente do processo. Confirmação disso são as seções de introdução da literatura sobre ECG para a formação docente (APPLEYARD \& MCLEAN, 2011; BLANKS, 2013; GUO, 2014; MCLEAN, COOK \& CROWE, 2006; ZONG, 2009), que frequentemente seguem a lógica que sua proposta é crucial para atender às demandas da globalização; A ECG é implementada pelos professores; Assim, preparar professores para ECG é imperativo. Essa afirmação está bem resumida nas três primeiras linhas do estudo de Zong (2009, p.617), feito sobre um projeto on-line da ECG para professores em formação:

[...] os educadores de professores hoje se deparam com uma responsabilidade urgente de transformar currículo e pedagogia para responder à demanda acelerando o crescimento da interdependência global em economia, tecnologia, política e cultura.

A literatura reflete, portanto, uma percepção sem demasiada evidência concreta de que a globalização exige a ECG, cujo sucesso, por sua vez, depende em grande parte de uma nova forma de preparação e de, talvez, ressocialização de professores (GÜNEL \& PEHLIVAN, 2016). Na nossa perspectiva, tal percepção é enganosamente simples e se traduz numa proposta circular: se a sociedade quer uma educação global cidadã, os professores precisam educar seus alunos para serem cidadãos globais, assim, os próprios professores também devem ser cidadãos globais. Várias propostas na literatura pedagógica ECG baseiam-se na premissa de que seus professores devam ser cidadãos globais em primeiro lugar (ver AN, 2014; APPLEYARD \& MCLEAN, 2011; BLANKS, 2013; BYKER, 2016; GUO, 2014; MCLEAN, COOK, \& CROWE, 2006). Como declara Byker (2016), “[...] o desenvolvimento da cidadania global entre os professores precisa começar antes mesmo de os professores assinarem seu primeiro contrato” (p. 264). Nesse modelo simples, os programas ECG precisam promover futuros professores para:

[...] estarem cientes da natureza global das questões sociais, cuidar das pessoas em lugares distantes, compreender a natureza da integração econômica global, apreciar a interconexão e interdependência dos povos, respeitar e proteger a diversidade cultural, lutar por justiça social para todos e para proteger o planeta Terra - lar de todos os seres humanos (ZHAO, 2010, p. 426).

Dado esse perfil que é idealizado, não surpreende que muitos estudos (AN, 2014; APPLEYARD \& MCLEAN, 2011; CARR, PLUIM \& HOWARD, 2014; MCLEAN, COOK \& CROWE, 2006; REIMER \& MCLEAN, 2009; ROBBINS, FRANCIS \& ELLIOT, 2003) tenham constatado que os docentes geralmente não dispõem de confiança e de habilidades 
pedagógicas para abordar o requerido pela ECG, embora considerem sua importância. A esse respeito, adotamos o conselho de Yemini (2017), ao afirmar que:

[...] os programas de formação de professores voltados para a promoção da ECG deveriam dar maior ênfase às dificuldades que os professores enfrentam frequentemente ao implementar ECG na sala de aula, em vez de limitar a discussão a seus muitos benefícios potenciais (p. 74).

Afinal, embora os professores mostrem uma melhor compreensão da ECG após capacitações (AN, 2014; APPLEYARD \& MCLEAN, 2011; BLANKS, 2013; BYKER, 2016; GUO, 2014; MCLEAN, COOK, \& CROWE, 2006), não significa necessariamente que eles se tornaram cidadãos globais mais engajados ou que o otimismo que esses programas aparentemente inspiram durará por muito tempo. Conforme anunciado no início deste trabalho compreensão e exercício prático são questões, embora importantes, diferentes.

O que não pode ser ignorado é que a formulação simples de um enquadramento idealizado da ECG gere uma narrativa potente que inspire pesquisadores e educadores. Diante disso e do quadro aqui desenhado, podemos dizer que a ECG torna-se uma versão contemporânea e renovada da já conhecida crença na potencial redenção pedagógica; em outras palavras, um processo escolar pelo qual a criança é capaz de se tornar um cidadão cosmopolita, cuja razão desencarnada, e tão somente ela, produz liberdade e inclusão (POPKEWITZ, 2008, 2009).

As supostas virtudes libertadoras do cosmopolitismo da educação para a cidadania global têm na visão do Popkewitz (2008), no entanto, o paradoxo de ser “[...] duplos gestos de esperança e medo, produzindo processos de exclusão com os da inclusão” (p. 301). Nas palavras de Andreotti (2015, p. 3), esse imaginário global assim divide a humanidade:

[...] entre aqueles que se percebem como detentores de conhecimento, trabalhadores esforçados, solucionadores de problemas mundiais, distribuidores de direitos, líderes globais; e aqueles que são percebidos como (e muitas vezes percebem suas culturas como) sem conhecimento, descontraídos, criadores de problemas, dependentes de ajuda e seguidores globais em sua jornada rumo ao objetivo indiscutível do desenvolvimento.

Não surpreende, portanto, que alguns comecem a considerar o cosmopolitismo como uma forma de capital $^{11}$ (MARSHALL, 2011; VEUGELERS, 2011; WEENINK, 2008) e, assim, uma tendência a ser incorporada como uma forma de expandir a comercialização de oportunidades educacionais, especialmente entre alguns, como escolas internacionais e programas educacionais que usam a ECG cosmopolita como parte de sua marca e sinal de distinção social (GARDNER-MCTAGGART, 2016; RESNIK, 2009, 2012). Por exemplo, a crescente expansão do currículo do International Baccalaureate (IB) no Sul Global, que, de acordo com Gardner-McTaggart (2016), ocorreu “[...] não por razões de equidade, ou cidadania global, mas em busca de vantagem relativa” (p. 1). Segundo o autor, a principal vantagem que as escolas do IB oferecem está relacionada ao capital cultural e simbólico que 
elas conferem, pois produz um claro senso de distinção e de privilégio (GARDNERMCTAGGART, 2016) ${ }^{12}$.

\section{Movimento Global de Reformas Educativas e Formação de Professores}

Conforme exposto até aqui, é importante entender o movimento que caminha em direção à expansão dos programas de ECG dentro do profundo impacto econômico da globalização nas práticas que favorecem o livre comércio, o investimento estrangeiro, a iniciativa privada e o comércio liberalizado que prevalecem e mudaram o tipo de trabalhadores demandados (STROMQUIST \& MONKMAN, 2000, p. 04).

A mudança no tipo de trabalhador necessário para os mercados globais influenciou as políticas, currículos e práticas educacionais, incluindo aquelas relacionadas à ECG (LINDGARD, et al, 2013). De fato, preparar os alunos com os conhecimentos, habilidades e competências necessários para competir em uma economia cada vez mais global também é uma intenção declarada por muitos programas da ECG (EVANS, 2009). Como Arnold (2014, p.35) afirma, a proposta da ECG “[...] é frequentemente empregada com o objetivo de produzir uma geração de jovens equipada com as habilidades transculturais necessárias para ter sucesso em um mercado global”, formando, assim, “[...] uma geração de líderes em desenvolvimento”, foco também destacado pelos estudos de Dicicco (2016).

Essa conexão entre mudanças educativas e as supostas demandas do mercado global não é independente do fato de que a ECG foi amplamente fomentada por organizações econômicas internacionais, como a Organização para Cooperação e Desenvolvimento Econômico (OCDE). Efetivamente, a atenção atual na ECG não é o resultado de demandas de professores ou de debates públicos sobre as funções e os objetivos da educação nacional, nem mesmo foi iniciada por países em desenvolvimento ou por grupos excluídos das formas tradicionais de cidadania, como minorias sociais, refugiados ou migrantes. Pelo contrário, a ECG foi iniciada em países como a Grã-Bretanha e os EUA ${ }^{13}$ e adotada por organizações internacionais que a expandiram rapidamente em todo o mundo. Algumas dessas organizações transnacionais que impulsionaram a ECG são instituições estritamente econômicas. Um dos mais relevantes é a OCDE, que também tem sido chamada de "Ministério Mundial da Educação" (PRIMAVERA, 2015, p. 64). A OCDE coloca em um mesmo nível, princípios como a "competitividade econômica”, a "competência global”, "habilidades do século XXI" e "habilidades de cidadania global” (NILSSON, 2015). Neste sentido, a literatura analisada apresenta uma ECG concentrada em preparar os alunos para serem cidadãos globais bem-sucedidos e aprendentes ao longo da vida, com uma alta dose de flexibilidade em conhecimentos e habilidades.

Em seu já anunciado paradoxo fundado no objetivo de constituir um sujeito globalmente educado, o discurso da ECG foi amplamente moldado e expandido por políticas neoliberais que se distanciam de discursos sobre a busca pela solidariedade global, sustentabilidade ou alfabetização transcultural (ANDREOTTI \& de SOUZA, 2012; ARNOLD, 2014; DICICCO, 2016; MYERS, 2016): 
Paradoxalmente, o neoliberalismo, ao lado de sua crítica às consequências mortais da "intrusão do Estado" na vida do indivíduo, provocou, não obstante, a invenção e/ou o emprego de toda uma série de formas organizacionais e métodos técnicos, a fim de estender o campo no qual um certo tipo de liberdade econômica pode ser praticado na forma de autonomia pessoal, empreendimento e escolha (BARRY, OSBORNE e ROSE, 1996, p. 10).

No centro do modelo do MGRE está, portanto, a ideia de que a melhor forma de acomodar as instituições educacionais à lógica comercial e aos mercados educativos. Os defensores dos MGRE acreditam fortemente no desempenho inerentemente superior do setor privado com fins lucrativos, mas eles não podem avançar sem o apoio de uma forte intervenção estatal (HURSH, 2001). Dentro da hegemonia neoliberal que conforma o MGRE, o foco e as agendas mudaram para programas de reforma escolar conduzidos e avaliados financeiramente, com consequências diretas para a formação de professores. Essas agendas estão centradas no aumento da responsabilidade e na eficiência das escolas através de processos de privatização/comercialização e terceirização de serviços educacionais; na expansão de plataformas online e digitais com o objetivo de gerar - e nesse contexto esse verbo é mais apropriado do que o verbo formar - professores e alunos com espírito empreendedor e senso de responsabilidade educacional individualizada. No caso específico da formação de professores, com o desenvolvimento de caminhos alternativos para a certificação de professores e o aumento do uso de currículos e pacotes educacionais "globalmente" orientados e comercializados.

Tal reforma pedagógica é entendida principalmente como um processo de produção de sistemas mais efetivos de recompensas e de punições para promover um mercado educacional baseado no mérito, ao mesmo tempo em que, por essa configuração, transforma objetivos, bases epistemológicas e métodos e procedimentos dos sistemas escolares públicos. De certa forma, essa é novamente uma transposição simples e poderosa de lógicas do mercado para o setor público. Como vimos, isso permite que professores e alunos sejam responsabilizados e que suas eficiências sejam medidas por avaliações educacionais internacionais de larga escala, afastando-os, assim, de suas condições de sujeitos; mais ainda, de suas especificidades, sobretudo socioeconômicas, historicamente constituídas e, sabemos, singulares a cada povo, muitas vezes dentro de uma mesma nação ${ }^{14}$.

Em instituições de formação de professores, o MGRE pode ser detectado na relação que as propostas sobre ECG têm com as demandas de educação do mercado global; a cultura de padronização e de responsabilização de seus atores; e a internacionalização da formação de professores. Embora a relação entre educação e crescimento econômico seja bastante incerta (RAMIREZ, LUO, SCHOFER \& MEYER, 2006), as demandas do mercado global, conforme o modelo aqui questionado, subordinou as instituições de formação de professores. A causa da competição em uma economia cada vez mais globalizada tornou-se, por sua vez, o núcleo da formação de professores nos dias de hoje ${ }^{15}$. 
"Flexibilidade”, “aprendizagem permanente”, "habilidades de TI” e “criatividade” compõem juntos um mantra de numerosos discursos de formação de professores (BATES, 2008; BERNSTEIN, 2000). Isso faz sentido em um contexto no qual a solução de problemas, a criatividade, a flexibilidade e a familiaridade com novas tecnologias são as qualidades que os empregadores desejam de candidatos competitivos (STEWART, 2007; WAGNER, 2008). Nessa esteira, e como consequência, os professores, passando por tal processo, teriam a tarefa de desenvolver a capacidade de treinar continuamente seus alunos para responder à instabilidade dos mercados e da tecnologia. Ao usar tais slogans, a ideia de que educação e formação de professores têm que servir às demandas econômicas se torna cada vez mais aceita socialmente. Além disso, a noção de que os futuros cidadãos terão que estar aptos a trabalhar em uma economia global em constante mudança, caracterizada pelo livre comércio se torna uma espécie de “mantra” (ARNOLD, 2014; NILSSON, 2015).

Esse modelo de economia política, naturaliza a redução das responsabilidades dos Estados com o bem-estar de seus cidadãos (STIGLITZ, 2012). Como Arnold (2014, p.5) astutamente observa, “[...] ao visionar a economia como um dado, os governos são absolvidos da responsabilidade na regulação da economia e na mitigação das desigualdades estruturais entre as populações”.

Esse modelo de formação de professores focado no treinamento para um mercado essencialmente privatizado nos direciona para uma crescente preocupação com a eficácia do ensino e com seu resultado, ou seja, um regime de padrões e de responsabilidade voltados para tornar professores e estudantes economicamente competitivos (SLEETER, 2008; TONNA, 2007). De fato, os ecos do imperativo global para a formação de professores ressoam em muitos documentos de padrões para a formação docente, frequentemente com um claro viés econômico. Por sua vez, esses padrões servem como parâmetros para avaliações internacionais que acabam redefinindo a perspectiva global da formação de professores (AYDAROVA \& MARQUARDT, 2016).

Neste contexto, também não é surpreendente que grandes esforços tenham sido destinados a desenvolver padrões e formas de medição da ECG, como demonstram, por exemplo, relatórios da OCDE (2016) e da UNESCO (2013). Alguns desses esforços foram promovidos diretamente por organizações econômicas internacionais. Por exemplo, a decisão da OCDE de incluir competências globais na rodada de 2018 do Programa Internacional de Avaliação de Estudantes (PISA), que teve um grande impacto sobre como a ECG é conceituada a ponto de "[...] a OCDE posicionar a ECG dentro de uma estrutura econômica de competitividade global” (NILSSON, 2015, p 2).

Cabe ainda ressaltar que as decisões adotadas pelos governos a esse respeito parecem seguir a mesma direção. Depois de estudar os padrões de cidadania global de dez estados dos EUA, Blevins (2011, p.157) concluiu que eles estavam mais focados na competitividade econômica global: “[....] os padrões de cidadania global foram desenvolvidos na tentativa de preparar adequadamente os alunos para o mundo cada vez mais globalizado do trabalho”.

A essas configurações somamos as faculdades e os cursos de formação de professores, que experimentaram um processo de internacionalização em todo o mundo nas últimas décadas (ACEDO, 2012; BÉGIN-CAOUETTE, 2012; LOW \& LEE, 2012; 
LUGOVTSOVA, KRASNOVA e TORHOVA, 2012; OLMEDO \& HARBON, 2010). Se esses esforços de internacionalização forem examinados criticamente, talvez não seja difícil encontrar valores de mercado globais em sua base (AYDAROVA \& MARQUARDT, 2016). Certamente, a internacionalização da formação de professores não pode ser entendida sem considerar a tendência neoliberal de promover um modelo de negócios na educação.

Como resposta às pressões globais de competição econômica, vários programas de formação de professores introduziram perspectivas internacionais em conteúdos curriculares e promoveram experiências de estudo e ensino no exterior (LARSEN, 2016). Além disso, muitos países de língua não inglesa usam cada vez mais o inglês como meio de instrução no ensino superior como uma manifestação das demandas do mercado global (LARSEN, 2016) que posicionam o inglês como língua franca com capital cultural e simbólico (BOURDIEU, 1991).

Nesse cenário, a cidadania global é frequentemente enquadrada como um capital cultural ou um status de prestígio obtido através da familiarização com outras línguas e culturas (WEENINK, 2008; ZEMACH-BERSIN, 2012). As oportunidades mais óbvias para esse engajamento surgem em programas de estudo no exterior, muitas vezes considerados como uma referência da educação intercultural e da cidadania global (DUNN, DOTSON, CROSS, KESNER e LUNDAHL, 2014; WILSON \& FLOURNOY, 2007). Sua expansão, somada a outras iniciativas internacionais, como escolas internacionais, longe de ser um processo para alcançar a justiça global, funciona como uma ferramenta para promover um tipo de cidadania global baseada na competitividade laboral (GARDNER-MCTAGGART, 2016; RESNIK, 2009, 2012). A cidadania global torna-se, portanto, uma identidade elitista e uma forma de distinção social (VEUGELERS, 2011; WEENINK, 2008), que a caracteriza como excludente.

\section{Podemos pensar em modelos não redentores ou consumistas na Educação para uma Cidadania Global?}

O otimismo e o pessimismo tornaram-se sinônimos da confiança do consumidor ou da falta dela. Ideias radicais sobre um mundo diferente tornaram-se quase literalmente impensáveis. As expectativas do que nós, como sociedade, podemos alcançar, foram drasticamente desgastadas, deixando-nos com a dura e fria verdade de que, sem a utopia, tudo o que resta é uma tecnocracia (BREGMAN, 2017, p. 15).

A reflexão de Bregman capta muito bem a tensão e os desafios apresentados pela ECG para a formação docente. Como observamos na introdução, encontramos indícios de otimismo e potencial de transformação real em propostas de ECG não romantizadas. Também permanecemos preocupados com o acoplamento dos modelos redentores dominantes de ECG que, na maioria dos casos, emprestam involuntariamente seus ideais sem muita reflexão para a promoção de MGRE nas quais o vencedor leva tudo. 
Não existe, em tais modelos, uma aliança formal ou explícita entre os setores que estão promovendo - e estão lucrando com - as ideias de privatização da educação e aqueles que entusiasticamente adotam visões românticas da educação para a cidadania global. O principal desafio, como o entendemos, é de manter os impulsos de transformação dos projetos educacionais globais cosmopolitas radicais, evitando, assim, a tentação de reduzi-los a boas listas de ações cidadãs, projetos normativos abstratos de cosmopolitismo light ou abordagens pedagógicas tecnocráticas sem conexões com a experiências dos alunos reais.

Como identificar um programa ECG contaminados pelos ideais dos MGRE? Infelizmente, não temos um procedimento simples e preventivo, mas acreditamos que qualquer programa da ECG que ignora intencionalmente os conflitos locais e globais, as desigualdades sociais e econômicas e descarta as complexidades, as controvérsias e as dinâmicas de opressão racial, sexual e linguísticas (entre outras), não são apenas contaminados pelo MGRE, mas também pedagogicamente negativos.

Os programas pedagógicos ECG só podem evitar servir aos interesses MGRE, na medida em que se posicionarem ceticamente em relação aos discursos empreendedores - e redentores - que moldam algumas propostas da ECG. Defendemos, portanto, uma ECG que renuncie às narrativas redentoras, que conforme aqui elucidado, esvazia, em suas propostas, os sujeitos de suas subjetividades. Somado a isso, que renuncie à idealização romântica da profissão docente, bem como a noção de que mais educação é a solução para os problemas globais urgentes.

Também defendemos a rejeição de qualquer visão do professor em formação como um “[...] receptor passivo de uma lista de 'bons comportamentos cosmopolitas”” (RIZVI \& BEECH, 2017, p. 128). Concordamos com Rizvi e Beech (2017) que visões normativas abstratas do cosmopolitismo acabam favorecendo práticas pedagógicas de teconocráticas, desconectadas das experiências cotidianas dos alunos. Tais renúncias implicam, também, em prestar mais atenção em como os programas realmente realizam a ECG e, novamente, não apenas sob este rótulo, e não em como devem fazê-lo.

A literatura revisada e dominada por programas pedagógicos de cidadania global com muitas - e aparentemente inofensivas - ideias românticas sobre solidariedades globais livres de conflitos não podem ser sustentadas sem uma insistência complementar da supremacia da escolha individual e da competição como a única resposta racional aos desafios globais. Esses tipos de programas provavelmente oferecerão uma proposta de ECG voltado para consumidores que deliberadamente esperam silenciosamente e talvez confortavelmente como resposta às crescentes desigualdades educacionais nos níveis local e global.

Uma nova forma de ECG exige uma tensão constante entre o imaginário utópico radical e uma ambição pedagógica de experimentação orientada a reafirmar os princípios da igualdade, diversidade e diferenças humanas, trabalhando para a democratização do acesso e para a permanência educacional, com oportunidades de aprendizagem mais inclusivas para todos os estudantes. Uma nova forma de ECG só pode ser um território pedagógico ainda desconhecido, com ideias, propostas, práticas que inevitavelmente se relacionaram em maior o menor medida com ferramentas antigas que foram desenvolvidos para prospectar o território de educação para a cidadania com foco nacional. Vale a pena parafrasear o que 
John Maynard Keynes (1937) disse, no prefácio de A Teoria Geral do Emprego, do Juro e da Moeda: a dificuldade reside não nas novas ideias, mas em fugir das antigas. Essa é a nossa humilde ambição. Não podemos fornecer um novo mapa pedagógico com uma rota detalhada para substituir a antiga cartografia redentora de muitas propostas de educação para a cidadania, mas queremos fazer parte da jornada, compartilhar a exploração, contribuir para uma melhor compreensão das possíveis rachaduras e acidentes, bem como o entusiasmo pela descoberta de melhores pedagogias, mais inclusivas, menos discriminadoras, engajadas com o bem público e -por que não? - mais alegres. Embora não resolva todos os problemas que afetam a formação docente, acreditamos que propostas diversas de educação cidadã críticas e não redentoras possam contribuir à produção de espaços para reflexões, intervenções e colaborações pedagógicas que encorajem os professores a ir além dos sonhos nostálgicos de escolaridade nacionalista romantizada ou os torturantes modelos neoliberais.

\section{Notas}

1. Por esta razão, alguns estudiosos defendem o que eles chamam de “cidadania múltipla” (HELD, 1997, 2016; URRY, 1998), o que implica o reconhecimento da pluralidade de níveis onde o engajamento cidadão pode acontecer: o estado, o local, o supra-estado e/ou nível transnacional.

2. Veja ARCHIBUGI \& HELD, 2011; BENHABIB, 2005; BENHABIB ET AL., 2006.

3. A filósofa política Seyla Benhabib (2005), uma das estudiosas mais influentes no campo, conceitua que as sociedades contemporâneas estão presenciando processos de desagregação da cidadania, porque os três pilares da cidadania nacional estão se tornando "desagregados”. Benhabib observa que esses pilares (a identidade coletiva, os privilégios de pertencer à identidade política de um Estado-nação e o direito a direitos sociais e benefícios de ser atribuído a um determinado Estado-nação) não estão mais integrados a um construto consistente e coerente (BENHABIB, 2005; 2007).

4. Ver, por exemplo, BLANKS, 2013; GUO, 2014; MCLEAN, COOK, \& CROWE, 2006; ZONG, 2009.

5. Outros associam a ECG à conexão entre globalização e educação (DOLBY \& RAHMAN, 2008; SPRING, 2008) ou com diferentes concepções de cidadania (KNIGHT ABOWITZ \& HARNISH, 2006).

6. Essa ideia de atores humanos como seres puramente conscientes tem sido largamente questionada por cientistas cognitivos como Ariely, (2008), Damasio, (2012); Kahneman, (2012), Lakoff, (2008) e por estudiosos sociais como Bourdieu (2007); Foucault, (2002) e Giddens (1995). Embora esses estudiosos apresentem pontos de partida, métodos e conclusões muito diferentes, todos eles apontam para as limitações dos modelos que explicam a "racionalidade” em termos de exercícios mentais sistematicamente conscientes, autorregulados e desencarnados.

7. Ver Abdi \& Shultz, 2008; Bennett, 2008; Carr \& Porfilio, 2012; Davies, Evans \& Reid, 2005 Dower, 2003; Evans, Ingram, Macdonald, \& Weber, 2009; Hébert, 2010; Hicks, 2003; Kerr, 2002; Mundy, Manion, Masemann E Haggerty, 2007; Oxley E Morris, 2013; Peters, Blee E Britton, 2008; Pike, 2000; Shultz, 2007.

8. Veja An, 2014; Appleyard E Mclean, 2011; Blanks, 2013; Byker, 2016; Guo, 2014; Mclean, Cook, \& Crowe, 2006.

9. Certamente, os debates sobre sua definição têm sido prolíficos e as tipologias são abundantes: uma educação de cidadania global leve e crítica (ANDREOTTI, 2006); abordagens neoliberal, radical e transformacional (SHULTZ, 2007); cidadania global aberta, moral e sociopolítica (VEUGELERS, 2011); abordagens técnico-econômicas e de justiça social (MARSHALL, 2011); tipos cosmopolitas e de defesa da cidadania global (OXLEY \& MORRIS, 2013).

10. Dado esses propósitos ambiciosos, não surpreendentemente, muitos autores têm criticado que os ideais da ECG não se traduzem em mudanças estruturais reais (MYERS, 2006, 2016; RAPOPORT, 2009, 2015). Embora muitos documentos curriculares possam adotar metas globais, eles, no entanto, estão firmemente enquadrados dentro dos limites de cada currículo e padrões nacionais-estaduais. Conforme Myers (2016) conclui sua análise comparativa, “[...] a estratégia de internacionalizar o currículo com discursos globais para a educação para a cidadania raramente tem desafiado o paradigma nacional dominante” (p. 8). Parece que, em termos de sua institucionalização, enquanto o ECG como campo se desenvolveu muito nos últimos anos, ainda há um longo caminho a ser consolidado (GAUDELLI, 2016). 
11. Weenink (2008) define o capital cosmopolita como “[...] uma propensão a engajar-se na arena social globalizante. [Isso] compreende predisposições e competências corporais e mentais (savoir faire), que ajudam a se engajar com confiança em tais arenas. Além disso, proporciona uma vantagem competitiva, uma vantagem inicial em relação aos concorrentes. As pessoas acumulam, implantam e exibem capital cosmopolita enquanto vivem no exterior por algum tempo, visitam e hospedam amigos de diferentes nacionalidades, participam de reuniões frequentemente para um público internacional, mantêm um círculo globalmente disperso de amigos ou parentes, leem livros, revistas e periódicos que alcance uma audiência global e possuem um domínio quase nativo do inglês e pelo menos uma outra língua”(p. 1092).

12. É importante considerar que 76\% das escolas IB estão em países anglo-saxônicos, mas a organização também está expandindo no Sul Global (IBO, 2013). De acordo com Hayden (2011), as escolas internacionais “[...] emergiram rapidamente como um meio de restauração não apenas para as classes profissionais globalmente móveis, mas também para as elites nacionais com vantagem socioeconômica para as quais uma forma de educação em inglês é percebida como concessão de vantagem adicional” (p. 18).

13. De acordo com o estudo feito por Parmenter (2011), 56\% dos 250 principais artigos relacionados ao ECG no WorldCat eram de instituições dos EUA, 18\% de instituições do Reino Unido, 6\% de instituições australianas e 5\% de instituições canadenses (p. 370).

14. O cenário acima elencado não é simplesmente um processo de medição e comparação, mas algo que afeta e modifica o que os programas de avaliação e responsabilização medem, impulsionados pela ideia reducionista de que tudo é uma questão de contabilidade e que as únicas lições educacionais que valem a pena serem ensinadas são aquelas que podem ser contadas e medidas nos exames internacionais.

15. Ver as avaliações críticas de Apple (2011), Bates (2008), Sleeter (2008), Romero e Luis (2007) e Tonna (2007).

\section{Referências}

ABDI, A., \& SHULTZ, L. (Eds.). Educating for human rights and global citizenship. Albany: State University of New York Press, 2008.

ACEDO, C. Internationalization of teacher education. Prospects, 42, 2012. p. 1-3.

AN, S. Preparing elementary teachers as global citizenship educators. Journal of Education, 194(2), 2014. p. 25-38.

ANDREOTTI, V. Soft versus critical global citizenship education, Policy \& Practice: A Development Education Review, 3, 2006. p. 40-51.

Global citizenship education otherwise: pedagogical and theoretical insights. In Ali Abdi, Lynette Shultz, and Tashika Pillay (eds.), Decolonizing Global Citizenship Education (pp. 221-230). Rotterdam: Sense Publishers, 2015. p. 221-230.

; DE SOUZA, L. M. T. M. Postcolonial perspectives on global citizenship education (Eds.). New York, NY: Routledge, 2012.

APPLE, M. W. Global crises, social justice, and teacher education. Journal of Teacher Education, 62(2), 2011. p. 222-234.

APPLEYARD, N.; MCLEAN, L. R. Expecting the exceptional: Pre-service professional development in global citizenship education. International Journal of Progressive Education, 7(2), 2011. p. 6-32.

ARCHIBUGI, D.; HELD, D. Cosmopolitan democracy: Paths and agents. Ethics \& International Affairs, 25(4), 2011. p. 433-461.

ARNOLD, E. Cultivating Global Citizens: Global Citizenship Education in a Moment of Neoliberal Globalization. (Masters thesis). Simon Fraser University, 2014.

AYDAROVA, E., \& MARQUARDT, S. K. The Global Imperative for Teacher Education: Opportunities for Comparative and International Education. Forum for International Research in Education, 3(1), 2016, p. 23-40.

BATES, R. Teacher education in a global context: Towards a defensible theory of teacher education. Journal of Education for Teaching, 34(4), 2008. p. 277-293. 
BAUMAN, Z. The individualized society. Cambridge, England: Polity Press, 2001.

BÉGIN-CAOUETTE, O. The internationalization of in-service teacher training in Québec cégeps and their foreign partners: An institutional perspective. Prospects, 42(1), 2012. p. 91-112.

BENHABIB, S. The rights of others: Aliens, residents, and citizens. Cambridge University Press, v. 5, 2004. Crises of the republic: Transformations of state sovereignty and the prospects of democratic citizenship. Justice, Governance, Cosmopolitanism, and the Politics of Difference - Reconfigurations in a Transnational World, Humboldt-Universitat zu Berlin, 2005. p. 45-78.

Democratic exclusions and democratic iterations, dilemmas of 'just membership' and prospects of cosmopolitan federalism. European Journal of Political Theory, 6, 2007. p. 445.

; WALDRON, J.; HONNIG, B.; KYMLICKA, W.; POST, R. Another cosmopolitanism. Oxford: Oxford University Press, 2006.

BENNETT, W. L. Changing citizenship in the digital age. In W. L. Bennett (Ed.), Civic life online: Learning how digital media can engage youth (pp. 1-24). Cambridge, MA: The MIT Press, 2008.

BERNSTEIN, B. Pedagogy, symbolic control and identity: Theory, research, critique. Oxford: Rowman \& Littlefield, 2000.

BLANKS, D. E. Nobel women: Drama pedagogy for global citizenship education. Global Partners in Education Journal, 3(1), 2013. p. 3-21.

BLEVINS, D. (2011). New Directions in Citizenship Education: Globalization, State Standards and an Ethical/Critical Social Studies Curriculum (Doctoral Dissertation), 2011. Disponível em: $<$ http://rave.ohiolink.edu/etdc/view?acc_num=osu1321839149>..

BOURDIEU, P. Language and symbolic power. Cambridge: Polity Press, 1991.

BYKER, E. J. Developing global citizenship consciousness: Case studies of critical cosmopolitan theory. Journal of Research in Curriculum \& Instruction, 20(3), 2016. p. 264-275.

CARR, P. R.; PORFILIO, B. J. (Eds.). Educating for peace in a time of 'permanent war': Are schools part of the solution or the problem? New York, NY: Routledge, 2012.

CAMICIA, S. P.; FRANKLIN, B. M. What type of global community and citizenship? Tangled discourses of neoliberalism and critical democracy in curriculum and its reform. Globalisation, Societies and Education, 9(3-4), 2011. p. 311-322.

CARNOY, M.; ELMORE, R.; SISKIN, L. (Eds.) The new accountability: High schools and high- stakes testing. New York, NY: Routledge-Falmer, 2003.

CARR, P. R.; PLUIM, G.; HOWARD, L. Linking global citizenship education and education for democracy through social justice: What can we learn from the perspectives of teacher-education candidates. Journal of Global Citizenship \& Equity Education, v. 4, n. 1, 2014. p. 1-21.

DALE, R.; ROBERTSON, S. L. New arenas of global governance and international organisations: Reflections and directions. In K. A. Martens, A. Rusconi, \& K. Leuze (Eds.), New arenas of education governance: The impact of international organizations and markets on educational policy making. New York, NY: Palgrave Macmillan, 2007. p. 217-228.

DAVIES, I.; EVANS, M.; REID, A. Globalising citizenship education? A critique of "global education" and "citizenship education". British Journal of Educational Studies, v.53, n. 1, 2005. p. 66-89.

DAVIES, L. Global citizenship: abstraction or framework for action? Educational Review, v. 58, n. 1, 2006. p. 5-25.

DE RIVERA, J.; CARSON, H. A. Cultivating a Global Identity. Journal of Social and Political Psychology, v. 3, n. 2, 2015. p. 310-330. 
DICICCO, M. Global citizenship education within a context of accountability and 21st century skills: The case of Olympus High School. Education Policy Analysis Archives, v. 24, n. 57, 2016. Disponível em: $<$ http://dx.doi.org/10.14507/epaa.24.2364>.

DILL, J. S. The longings and limits of global citizenship education: the moral pedagogy of schooling in a cosmopolitan age. New York, NY: Routledge, 2015.

DOLBY, N., \& RAHMAN, A. Research in international education. Review of Educational Research, v. 78, n. 3, 2008. p. 676-726.

DOWER, N. An introduction to global citizenship. Edinburgh, Scotland: Edinburgh University Press, 2003.

DUCKWORTH, R. L.; LEVY, L. W.; LEVY, J. Present and future teachers of the world's children How internationally-minded are they? Journal of Research in International Education, v. 4, n. 3, 2005. p. 279311.

DUNN, A. H.; DOTSON, E. K.; CROSS, S. B.; KESNER, J.; LUNDAHL, B. Reconsidering the local after a transformative global experience: A comparison of two study abroad programs for preservice teachers. Action in Teacher Education, v. 36, 2014. p. 283-304.

EIDOO, S., Ingram, L. A., MACDONALD, A., NABAVI, M., PASHBY, K., \& STILLE, S. "Through the kaleidoscope": Intersections between theoretical perspectives and classroom implications in Critical Global Citizenship Education. Canadian Journal of Education, v. 34, n. 4, 2011. p. 59-85.

ENSLIN, P. Education and democratic citizenship: In defence of cosmopolitanism. In M. Leicester, C. Modgil, \& S. Modgil (Eds.), Politics, education and citizenship. New York: Falmer Press, 2000. p. 149-150.

EVANS, M.; INGRAM, L. A.; MACDONALD, A.; WEBER, N. Mapping the "global dimension" of citizenship education in Canada: The complex interplay of theory, practice and context. Citizenship Teaching and Learning, v. 5, n. 2, 2009. p. 17-34.

EVANS, R. W. Schooling corporate citizens: how accountability reform has damaged civic education and undermined democracy. New York, NY: Routledge, 2015.

FISCHMAN, G. E., \& HAAS, E. Moving beyond idealistically narrow discourses in citizenship education. Policy Futures in Education, v. 12, n. 3, 2014. p. 387-402.

FISCHMAN, G. E., \& HAAS, E. Beyond Idealized Citizenship Education Embodied Cognition, Metaphors, and Democracy. Review of Research in Education, v. 36, n. 1, 2012. p. 169- 196.

GARDNER-MCTAGGART, A. International elite, or global citizens? Equity, distinction and power- the International Baccalaureate and the rise of the South. Globalisation, Societies and Education, v. 14, n. 1, 2016. p. 1-29.

GAUDELLI, W. Heuristics of global citizenship discourses towards curriculum enhancement. Journal of Curriculum Theorizing, v. 25, n. 1, 2009. p. 68-85.

. Global citizenship education: everyday transcendence. New York: Routledge, 2016.

GIMENO, P. Didáctica crítica y comunicación: un diálogo con Habermas y la Escuela de Frankfurt. Barcelona: Octaedro, 2009.

GRIFFITHS, R. Educational citizenship and independent learning. London: Jessica Kingsley, 1998.

GÜNEL, E.; PEHLIVAN, A. Pre-Service Social Studies Teachers’ Perception of Global Citizenship. Journal of Education and Future, v. 10, 2016. p. 51-69.

GUO, L. Preparing teachers to educate for 21st century global citizenship: Envisioning and enacting. Journal of Global Citizenship \& Equity Education, v. 4, n. 1, 2014. p. 1-23.

HAHN, C. L. Diversity and human rights in England and the UniEdP States. In A. Osler (Ed.), Teachers, human rights and diversity (pp. 23-34). London, UK: Trentham books, 2005.

HANDLER, R. Disciplinary adaptation and undergraduate desire: Anthropology and global development studies in the liberal arts curriculum. Cultural Anthropology, v. 28, n. 2, 2013. p. 181-203. 
HARTMAN, E.; KIELY R. A Critical Global Citizenship. In: GREEN, P.; JOHNSON, M. (Eds.), Crossing boundaries: tension and transformation in international service-learning. Sterling, VA: Stylus, 2014. p. 215-242.

HAYDEN, M. Transnational spaces of education: The growth of the international school sector. Globalisation, Societies and Education, v. 9, n. 2, 2011. p. 211-224.

HEATER, D. A History of Education for Citizenship. London, UK: Routledge Falmer, 2004.

HÉBERT, Y. Politics, citizenship education policy in twelve countries, and cosmopolitanism: a commentary. In: REID, A.; GILL, J.; SEARS, A. (Eds.), Globalization, the nation-state and the citizen. New York, NY: Routledge, 2010. p. 230-240.

HELD, D. La democracia y el orden global. Del estado moderno al gobierno cosmopolita. Barcelona, Spain: Paidós 1997.

Cosmopolitanism. Ideals and realities. Cambridge, UK: Polity Press, 2010.

Elements of a theory of global governance. Philosophy \& Social Criticism, v. 42, n. 9, 2016. p. 837-846.

HICKS, D. Thirty years of global education: A reminder of key principles and precedents. Educational Review, v. 55, n. 3, 2003. p. 265-275.

HURSH, D. Neoliberalism and the control of teachers, students, and learning: The rise of standards, standardization, and accountability. Cultural Logic, v. 4, n. 1, 2001. p. 4-1.

IBO. IB Fast Facts. IBO, 2013. Disponível em: <http://www.ibo.org/facts/fastfacts/> .

JEFFRESS, D. The "Me to We" social enterprise: global education as lifestyle brand. Critical Literacy: Theories and Practices, v. 6, n. 1, 2012. p. 18-30.

JORGENSON, S.; SHULTZ, L. Global citizenship education (ECG) in post-secondary institutions- What is protecEdP and what is hidden under the umbrella of ECG. Journal of Global Citizenship \& Equity Education, v. 2, n. 1, 2012. p. 1-22.

KERR, D. Citizenship education: an international comparison across sixteen countries. International Journal of Social Education, v. 17, n. 1, 2002. p. 1-15.

KEYNES, J. M. The General Theory of Employment, Interest and Money. London: Macmillan, 1936.

KNIGHT ABOWITZ, K. On the public and civic purposes of education. Educational Theory, v. 58, 2008. p. 357-376.

KNIGHT ABOWITZ, K.; HARNISH, J. Contemporary discourses of citizenship. Review of Educational Research, v. 74, n. 4, 2006. p. 653-690.

LARSEN, M. A. Globalisation and internationalisation of teacher education: a comparative case study of Canada and Greater China. Teaching Education, v. 27, n. 4, 2016. p. 396-409.

; FADEN, L. Supporting the growth of global citizenship educators. Brock Education, v. 17, 2008. p. 71-86.

LINGARD, B.; MARTINO, W.; REZAI-RASHTI, G. Testing regimes, accountabilities and education policy: Commensurate global and national developments. 2013.

LONGVIEW FOUNDATION. Teacher preparation for the global age: the imperative for change. 2018. Disponível em: <http://www.longviewfdn.org/122/teacher-preparation-for-the- global-age.html>.

LOW, E. L.; LEE, S. K. Bringing Singapore's teacher education beyond its shores. Educational Research for Policy and Practice, v. 11, n. 1, 2012. p. 43-51.

LUGOVTSOVA, A.; KRASNOVA, T.; TORHOVA, A. Internationalizing teacher education: the case of Belarus. Prospects, v. 42, 2012. p. 71-90. 
MARCUS, G. E. Sentimental Citizen: Emotion in Democratic Politics. Pennsylvania: The Pennsylvania State University Press, 2002.

MANNION, G.; BIESTA, G.; PRIESTLEY, M.; ROSS, H. The global dimension in education and education for global citizenship: Genealogy and critique. Globalisation, Societies and Education, v. 9, n. 3-4, 2011. p. 443-456.

MARSHALL, H. Instrumentalism, ideals and imaginaries: Theorising the contesEdP space of global citizenship education in schools. Globalisation, Societies and Education, v. 9, n. 3-4, 2011. p. 411-426.

MCLEAN, L. R.; COOK, S. A.; CROWE, T. Educating the Next Generation of Global Citizens through Teacher Education, One New Teacher at a Time. Canadian Social Studies, v. 40, n. 1, 2006.

MUNDY, K.; MANION, C.; MASEMANN, V.; HAGGERTY, M. Charting global education in Canada's elementary schools: Provincial, district, and school level perspectives. Toronto, ON: UNICEF Canada, 2007.

MYERS, J. P. Rethinking the social studies curriculum in the context of globalization- Education for global citizenship in the US. Theory \& Research in Social Education, v. 34, n. 3, 2006. p. 370-394.

Charting a democratic course for global citizenship education: Research directions and current challenges. Education Policy Analysis Archives, v. 24, n. 55, 2016. p. 1-19.

NIKOLAS, R.; BARRY, A.; OSBORNE, T.; ROSE, N. Governing “advanced” liberal democracies. Foucault and Political Reason. London: UCL Press, 1996. p. 37-64.

NILSSON, I. Constructing Global Citizenship Education: an analysis of OECD discourse on global competence. (Unpublished Paper). Department of Political Science, Lund University, 2015.

NODDINGS, N. Global citizenship: Promises and problems. In N. Noddings (Ed.), Educating citizens for global awareness. New York, NY: Teachers College Press, 2005. p. 1-21.

NUSSBAUM, M. C. Cultivating humanity: a classical defense of reform in liberal education. Cambridge, MA: Harvard University Press, 1997.

OCDE. Global competency for an inclusive world. Paris: OECD, 2016.

OLMEDO, I.; HARBON, L. Broadening our sights: Internationalizing teacher education for a global arena. Teaching Education, v. 21, n. 1, 2010. p. 75-88.

OSLER, A. Teachers, Human Rights and Diversity. London: Trentham Books, 2005.

OXLEY, L.; MORRIS, P. Global citizenship: A typology for distinguishing its multiple conceptions. British Journal of Educational Studies, v. 61, n. 3, 2013. p. 301-325.

PARKHOUSE, H.; TICHNOR-WAGNER, A.; CAIN, J. M.; GLAZIER, J. "You don't have to travel the world": accumulating experiences on the path toward globally competent teaching. Teaching Education, v. 27, n. 3, 2016. p. 1-19.

PARMENTER, L. Power and place in the discourse of global citizenship education. Globalisation, Societies and Education, v. 9, n. 3-4, 2011. p. 367-380.

PETERS, M.; BLEE, H.; BRITTON, A. Global citizenship education: Philosophy, theory and pedagogy. Rotterdam, The Netherlands: Sense Publishers, 2008.

PIKE, G. A tapestry in the making: The strands of global education. In T. Goldstein \& D. Selby (Eds.), Weaving connections: Educating for peace, social and environmental justice. Toronto, ON: Sumack Press, 2000. p. 218-241.

Global education. In J. Arthur, I. Davies \& C. Hahn (Eds.), The Sage handbook of education for citizenship and democracy. London: Sage, 2008. p. 468-480.

POPKEWITZ, T. S. Global education as a slogan system. Curriculum Inquiry, v. 10, n. 3, 1980. p. 303-316. Education sciences, schooling, and abjection: recognizing difference and the making of inequality?. South African Journal of Education, v. 28, n. 3, 2008. p. 301-319. 
El cosmopolitismo y la era de la reforma escolar. La ciencia, la educación y la construcción de la sociedad mediante la construcción de la infancia. Madrid: Morata, 2009.

RAMIREZ, F.; LUO, X.; SCHOFER, E.; MEYER, J. Student achievement and national economic growth. American Journal of Education, v. 113, n. 1, 2006. p. 1-29.

RAPOPORT, A. A forgotten concept: Global citizenship education and state social studies standards. Journal of Social Studies Research, v. 33, n. 1, 2009. p. 91-112.

We cannot teach what we don't know: Indiana teachers talk about global citizenship education. Education, Citizenship and Social Justice, v. 5, n. 3, 2010. p. 179-190.

Global citizenship education. Classroom teachers’ perspectives and approaches. In J. R. Harshman, T. Augustine, \& M. M. Merryfield (Eds.). Research in global citizenship education. Greensboro, NC: Information Age Publishing, 2015. p. 119-135.

REIMER, K.; MCLEAN, L. R. Conceptual clarity and connections: Global education and teacher candidates. Canadian Journal of Education, v. 32, n. 4, 2009. p. 903-926.

RESNIK, J. Multicultural education: good for business but not for the state? The IB curriculum and global capitalism. British Journal of Educational Studies, v. 57, n. 3, 2009. p. 217-244.

The denationalization of education and the expansion of the International Baccalaureate. Comparative Education Review, v. 56, n. 2, 2012. p. 248-269.

RIZVI, F.; BEECH, J. Global mobilities and the possibilities of a cosmopolitan curriculum, Curriculum Inquiry, v. 47, n. 1, 2017. p. 125-134.

ROBBINS, M.; FRANCIS, L. J.; ELLIOTT, E. Attitudes toward education for global citizenship among trainee teachers. Research in Education, v. 69, n. 1, 2003. p. 93-98.

ROBERTS, A. Global dimensions of schooling: Implications for internationalizing teacher education. Teacher Education Quarterly, v. 34, n. 1, 2007. p. 9-26.

ROBERTSON, S. L. What teachers need to know about the "Global Education Reform Movement” (or MGRE). In: LITTLE, G. (Ed.), Global education 'reform'. Building resistance and solidarity. Manifesto Press, 2015. p. 10-17.

ROBERTSON, S. L., \& DALE, R. Researching education in a globalising era: Beyond methodological nationalism, methodological statism, methodological educationism and spatial fetishism. In J. Resnik (Ed.), The production of educational knowledge in the global era. Rotterdam, Netherlands: Sense, 2008. p. 1932.

ROMERO, J.; ESTELLÉS, M. Educación para la ciudadanía y currículum: sus regímenes de verdad en perspectiva histórica. In: BORGHI, B.; GARCÍA, F. F.; MORENO, O. (Eds.), Novi cives: cittadini dall'infanzia in poi. Bologna: Pàtron Editore, 2015. p. 63-76.

; LUIS, A. ¿Sirven las políticas y prácticas de formación del profesorado para mejorar la educación? Una respuesta desde el análisis de la construcción social de la docencia. Archivos Analíticos de Políticas Educativas, v. 15, n. 19, 2007. Disponível em: <http://dx.doi.org/10.14507/epaa.v15n19.2007>.

SAHLBERG, P. Rethinking accountability in a knowledge society. Journal of Educational Change, v. 11, n. 1, 2010. p. 45-61.

SCHUGURENSKY, D. Citizenship learning for and through participatory democracy. In E. Pinnington \& D. Schugurensky (Eds.), Learning citizenship by practicing democracy: International initiatives and perspectives. Newcastle upon Tyne, England: Cambridge Scholarly Press, 2010. p. 1-19.

SHULTZ, L. Educating for global citizenship: Conflicting agendas and understandings. Alberta Journal of Educational Research, v. 53, n. 3, 2007. p. 248-258.

SLEETER, C. Equity, democracy, and neoliberal assaults on teacher education. Teaching and Teacher Education, v. 24, n. 8, 2008. p. 1947-1957. 
SPRING, J. Research on globalization and education. Review of Educational Research, v. 78, n. 2, 2008. p. 330-363.

Globalization of education: an introduction. New York, NY: Routledge, 2015.

STEWART, V. Becoming citizens of the world. Educational Leadership, v. 64, n. 7, 2007. p. 8-14.

STIGLITZ, J. The price of inequality. New York, NY: Norton \& Company, 2012.

STROMQUIST, N. P.; MONKMAN, K. Globalization and education: Integration and contestation across cultures. Lanham: Rowman \& Littlefield Education, 2014.

SU, F.; BULliVANT, A.; HOLT, V. Global Citizenship Education. In: Curtis, W., et al (eds), Education Studies: An Issues Based Approach. Exeter: Sage/Learning Matters, 2013. p. 231-244.

SUÁREZ-OROZCO, M. M. (Ed.). Learning in the global era: International perspectives on globalization and education. Berkeley, CA: University of California Press, 2007.

TONNA, M. A. Teacher education in a globalized age. Journal for Critical Education Policy Studies, v. 5, n. 1, 2007. p. 1-28.

TRILOKEKAR, R. D.; KUKAR, P. Disorienting experiences during study abroad: Reflections of pre-service teacher candidates. Teaching and Teacher Education, v. 27, n. 7, 2011. p. 1141-1150.

UNESCO. Global Citizenship Education: An Emerging Perspective. Outcome document of the Technical Consultation on Global Citizenship Education, 2013. Disponível em: $<$ http://www.unesco.org/new/en/education/resources/online-materials/singleview/news/unescos_seoul_consultation_deepens_understanding_of_global_citizenship_education/\#.UjxK SX-bFng>.

. Global Citizenship Education. Preparing learners for the challenges of the 21st century. Paris: UNESCO, 2014. Disponível em: <http://unesdoc.unesco.org/images/0022/002277/227729e.pdf>.

URRY, J. Globalisation and citizenship. Department of Sociology, University of Lancaster, UK, 1998. Disponível em: <http://www.lancs.ac.uk/fass/sociology/papers/urry-globalisation-and-citizenship.pdf>.

VEUGELERS, W. The moral and the political in global citizenship: Appreciating differences in education. Globalisation, Societies and Education, v. 9, n. 3-4, 2011. p. 473-485.

WAGNER, T. The global achievement gap. New York, NY: Basic Books, 2008.

WANG, C.; HOFFMAN, D. M. Are WE the world? A critical reflection on selfhood and global citizenship education. Education Policy Analysis Archives, v. 24, n. 56, 2016.

WEENINK, D. Cosmopolitanism as a Form of Capital: Parents Preparing Their Children for a Globalizing World. Sociology, v. 42, n. 6, 2008. p. 1089-1106.

WILSON, A.; FLOURNOY, M.A. Preparatory courses for student teaching abroad. In: CUSHNER, K; BRENNAN, S. (Eds.). Intercultural student teaching: a bridge to global competence. Plymouth, UK: Rowman \& Littlefield Education, 2007. p. 34-56.

YEMINI, M. Internationalization and Global Citizenship. Policy and Practice in Education. Palgrave Macmillan. 2017.

ZEMACH-BERSIN, T. Entitled to the World: The rhetoric of U.S. global citizenship education and study abroad. In: ANDREOTTI, V.; SOUZA, L. M. T. M. de (Eds.). Postcolonial perspectives on global citizenship education. New York: Routledge, 2012. p. 87-104.

ZHAO, Y. Preparing globally competent teachers: A new imperative for teacher education. Journal of Teacher Education, v. 61, n. 5, 2010. p. 422-431.

ZONG, G. Developing preservice teachers’ global understanding through computer-mediaEdP communication technology. Teaching and Teacher Education, v. 25, n. 5, 2009. p. 617-625. 


\section{Correspondência}

Gustavo E. Fischman é Professor da Arizona State University, Estados Unidos.

Email: fischman@asu.edu

Marta Estellés é professora da Universidade de Cantabria, Espanha.

Email: marta.estelles@unican.es

Texto publicado em Currículo sem Fronteiras com autorização dos autores. 